International Journal of Electronics and Communication Engineering and Technology (IJECET)

Volume 12, Issue 1, January-April 2021, pp. 8-19. Article ID: IJECET_12_01_002

Available online at https://iaeme.com/Home/issue/IJECET?Volume=12\&Issue=1

ISSN Print: 0976-6464 and ISSN Online: 0976-6472

DOI: 10.34218/IJECET.12.1.2021.002

\title{
INTENSIFICATION AND OPTICAL PENETRATION IN PLASMONIC THIN FILM SOLAR CELL BY NANO PARTICLE ON METAL DIELECTRIC SEMICONDUCTOR-PHOTONIC RESPONSE
}

Nabamitaa Roy*
Electronics and Communication Engineering, Narula Institute of Technology, Kolkata, India

Priya Mahto

Electronics and Communication Engineering, Narula Institute of Technology, Kolkata, India

\section{Samarpita Basu}

Electronics and Communication Engineering, Narula Institute of Technology, Kolkata, India

\section{Oudarjya Sen Sarma}

Electronics and Communication Engineering, Narula Institute of Technology, Kolkata, India

\section{Shreyasi Das}

Electronics and Communication Engineering, Narula Institute of Technology, Kolkata, India

\section{Dr. Saradindu Panda}

R\&D-Dean, Narula Institute of Technology, Kolkata, India

*Corresponding Author

\begin{abstract}
As of late, Plasmonic gives expanded interest that has been gotten as an approach to improvement in photonic reaction at optical termination at metal-dielectric interface. Its featured property is that nanostructure can handle optical fields because of the solid association of the metal-dielectric structure that permits episode photonic spread at the nano scale. In this examination, we research and reproduce the viability of different huge band gap dielectric materials like silicon nitride, aluminum oxide and silicon dioxide with different metallic nano particle and furthermore reenact limited time sensitive metal dielectric-semiconductor nanostructure and discover different field part for plasmonic improvement in type of photonic elimination in metal-dielectric
\end{abstract}


nanostructure explicitly for sun-based energy reaping system. Photovoltaic sunlightbased cell changes over phonic energy into electrical energy.

Keywords: absorption, photovoltaic sunlight, photonic spread, phonic energy, surface Plasmon.

Cite this Article: Nabamitaa Roy, Priya Mahto, Samarpita Basu, Oudarjya Sen Sarma, Shreyasi Das and Saradindu Panda, Intensification and Optical Penetration in Plasmonic thin Film Solar Cell by Nano Particle on Metal Dielectric Semiconductor-Photonic Response, International Journal of Electronics and Communication Engineering and Technology, 12(1), 2021, pp. 8-19. https://iaeme.com/Home/issue/IJECET?Volume=12\&Issue=1

\section{INTRODUCTION}

At the point when episode photonic energy is move similarly to wave vector of surface Plasmon, surface Plasmonic reverberation happens. The changes of wave vectors rely upon dielectric consistent of encompassing mode of nano metal. Currently, our attainable sun-based cell proficiency is approx. 16-18\%. In the sunlight based photovoltaic cell, if occurrence photonic energy is more than or equivalent to the band-hole energy, the sun-oriented energy is consumed and produces an electron-opening pair. Something else, here happen sub-band misfortunes. Plasmonic is a most up to date arising plan procedures that might be a potential method to progress in photonic assimilation in sun-based cell and improve ingestion by photonic dissipating because of metallic nano particles which are energized at surface Plasmonic reverberation. Sun oriented energy episode on the earth surface is around 1,650 TW/sec is a lot of conventional fuel sources ${ }^{[1]}$. Consequently, the sun-based energy has the benefit to create greater power. For this reason, an interest for enormous scope creation of sun powered cell has arisen financially higher than the joined force utilization by our reality is about 20TW utilizing Conventional-fuel sources. Consequently, the sun-based energy has the benefit to create greater power. For this reason, an interest for enormous scope creation of sun powered cell has arisen financially in 1954; the show of semiconductor-based sunlight-based cell is explored with beginning efficiency approx. $6 \%$ in Bell Laboratories ${ }^{[2]}$. After that to in any case now there are so many proposals has been coming for sunlight-based cell planning dependent on utilized materials, techniques to improve productivity $(\eta)$ and so on. Today market-driving monetarily accessible sun powered cell is glasslike Si PV sun-based cell with productivity up to $14.7 \%{ }^{[3]}$. Utilizing other plan method, like back surface field (BSF) gives further improvement at $15.5 \%$ and back neighborhood contact (RLC) improve effectiveness up to $20 \%{ }^{[3]}$. As the original Si sunlight-based cell has a few disadvantages and furthermore to bring down cost per watt interest, second and third era sun-oriented cell has been come which depend on slim film innovation and ease with adaptable substrates. From the start to introduce situation, the effectiveness of sunlight-based cell is too low and over $80 \%$ is a misfortune. In a sun-oriented cell, if the photonic energy is more noteworthy than the band gap energy, this photonic band is consumed and creates electron $\left(\mathrm{e}^{-}\right)$opening $(\mathrm{h})$ pair. In the event that photon energy is not exactly the band gap, there happens sub band misfortune. Plasmonic are one of freshest promising methods as it improves ingestion by photon dispersing utilizing metal nano particle energized at their surface Plasmon reverberation ${ }^{[4]}$. Utilizing metallic nano particle, the Raman dispersing is expanded and it additionally gives more photons that will be accessible to energize surface Plasmon therefore electrons to be energized and travel through the sun powered cell to produce a photocurrent. 


\section{BASIC SOLAR CELL}

Photovoltaic sunlight-based cell assimilates occurrence photonic energy converts to electrical energy and the activity is like PN intersection diode. In the event that the episode photon energy is more noteworthy than or equivalent to the band gap energy, at that point the electron-opening pair will be produced. Electrons are gathered to deliver power as in (Sagadevan, 2013). ${ }^{[5]}$

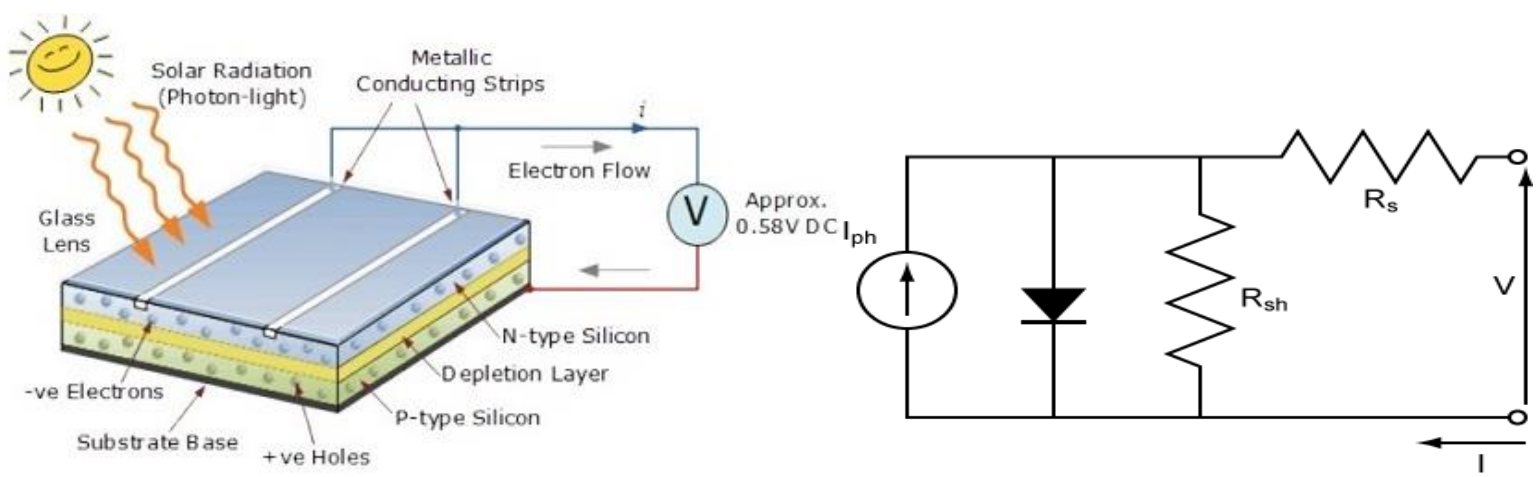

Figure 1 Basic Structure of Solar Cell \& Equivalent Circuit

Under brightening at sun-oriented cell, the produced photocurrent relies upon the occurrence light frequency and furthermore force relies upon quantum effectiveness which profoundly relies upon the assimilation coefficient of the material. So, the resultant current is the amount of light current and dull current. At the point when the sunlight-based cell is short circuited, the short out current $\mathrm{I}_{\mathrm{SC}}$ turns out to be light current $\mathrm{I}_{\mathrm{L}}$. At the point when the cell is at no heap condition, the potential will be most extreme and under a specific degree of light, the open circuit voltage $\mathrm{V}_{\mathrm{oc}}$, the fill factor FF is a proportion of the parity of the I-V trademark.

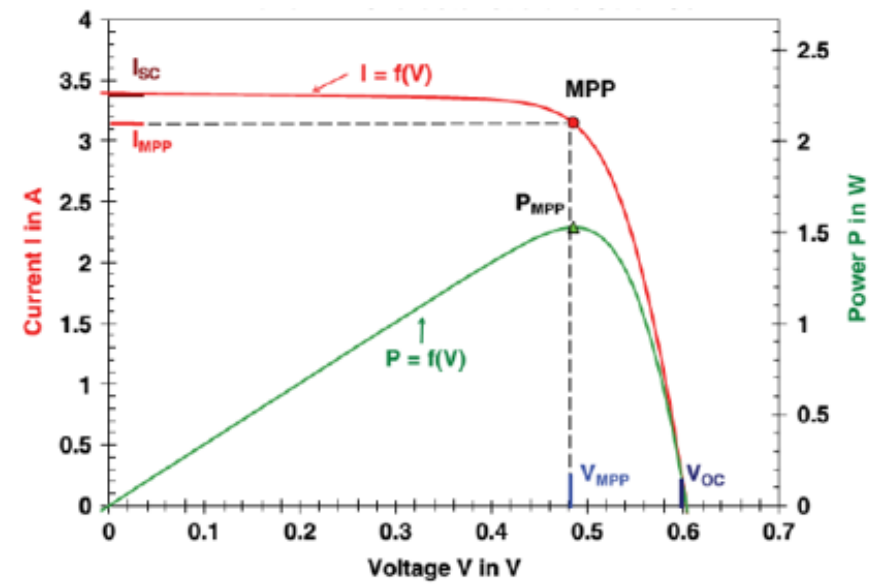

Figure 2 Solar characteristics I-V graph

\section{PLASMONIC SOLAR CELL}

Plasmon is a thick, wave in an electron gas that sways in a state of harmony. Plasmon exists predominantly in metals, where electrons are loosely bound to the molecule and allowed to meander. For the decision of metal, Nobel metals (gold, silver) are best for plasmonic reverberation. Plasmon is an aggregate wavering of electron cloud which sways in a state of harmony. The plasmonic sensation happens chiefly in metals, where valence electrons are delicately confined with parent atoms. Plasmons are two classes-limited surfaces Plasmon and engendering plasmon. The restricted surface plasmon happens due to the dipole and additionally multipole wavering of electron which sync at locally at the metal-dielectric interface. An ordinarily limited Plasmon presence in the electric field is appeared in Fig. 1. 


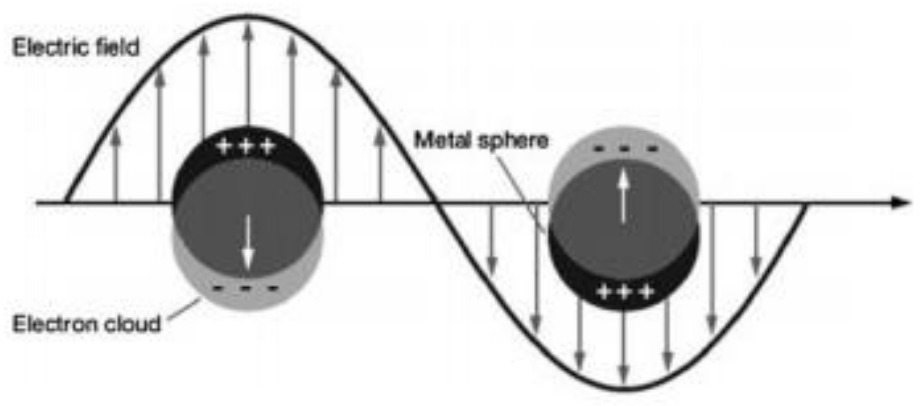

Figure 3 Localized surface Plasmonic fluctuation with nano-metal

Plasmonic impact for the most part happen in metal with valence electron, these electron cloud models are portrayed with the Jellium model ${ }^{[6]}$. From traditional mechanics, the presence of the uniform positive electric field, the electron thickness is moved to the x-bearing as in. So, nonattendance of electric fields, the electron thickness gets sped up by the field which is produced by the surface charges and furthermore they return back to unique position. Thus, they have encountered a force along the positive $\mathrm{x}$-hub and hence create an electric field of inverse sign. This cycle will be rehashed and brings about an aggregate swaying as in. By this way, the electrons will oscillate by the electric field due to the surface charges. Using classical theory applied to a single electron:

$$
m \frac{d^{2} x}{d t^{2}}=e E_{x}
$$

Where $E_{x}$ is electric field due to the surface charges.

\section{MODELING \& ANALYSIS}

From Gauss's theorem, it is considering that the electric field generated by a sheet carrying a surface charge nex will be $E_{x}=\frac{2 n e x}{2 \varepsilon_{0}}$. The collective oscillation of the electrons in the bulk and the plasma frequency are $\omega_{p}=\sqrt{\frac{n e^{2}}{m \varepsilon_{0}}}$.

In this study, we have considered metallic nano sphere, whose radius is very smaller than the incident wavelength. So, the generated field due to uniform polarization $\mathrm{P}_{\mathrm{x}}$ of the particle $E_{x}=-P_{x} / 3 \varepsilon_{0}$. So, the resonance frequency of the Plasmon in nano-sphere is given by:

$$
\omega_{s p}=\frac{\omega_{p}}{\sqrt{3}}=\sqrt{\frac{n e^{2}}{3 m \varepsilon_{0}}}
$$

The surface plasmon polarization is a class of surface waves which engender at the interface between a metal and a dielectric. For surface plasmon, respectable metals like Ag and Au are the most ideal decision whose dielectric steady is characterized by to the Drude model ${ }^{[6]}$ and additionally the dielectric consistent exceptionally relies upon occurrence recurrence:

$$
\varepsilon_{r}(\omega)=1-\frac{\omega_{p}^{2}}{\omega^{2}+i \gamma(\omega) \omega}
$$

\subsection{Dispersing and Assimilation by Metallic Nano Particles}

The assimilation upgrade in metallic nano particle into a sun-oriented cell happens because of light dispersing and close field convergence of light. Metal nano particles are solid disperses of light at frequencies close to the plasmon reverberation, which is because of an aggregate 
wavering of the conduction electrons in the metal. As indicated by Mie hypothesis, the dissipating and termination cross areas $(\sigma)$ are given by ${ }^{[10]}$ :

$$
\sigma_{\text {sca }}=\frac{\lambda^{2}}{2 \pi} \sum_{n=0}^{\alpha}(2 n+1)\left(\left|a_{n}\right|^{2}+\left|b_{n}\right|^{2}\right) \quad \sigma_{\text {ext }}=\frac{\lambda^{2}}{2 \pi} \sum_{n=0}^{\alpha}(2 n+1) \operatorname{Re}\left(a_{n}+b_{n}\right)
$$

Here $\varepsilon_{\mathrm{sp}}(\omega)$ is dielectric constant of metallic nano sphere and $\varepsilon_{\mathrm{em}}$ is dielectric constant of embedded medium. Full upgrade happens under the condition that $\left|\varepsilon_{\mathrm{sp}}(\omega)+2 \varepsilon_{\mathrm{em}}\right|$ is a base. The comparing proficiency $(\mathrm{Q})$ for dispersing, ingestion is given beneath:

$$
Q_{a b s}=\frac{8 \pi r}{\lambda} \operatorname{Im}\left\{\frac{\varepsilon_{s p}(\omega)-\varepsilon_{\mathrm{cm}}}{\varepsilon_{s p}(\omega)+2 \varepsilon_{\mathrm{cm}}}\right\}
$$

For a circular metallic nano sphere with volume $V=\frac{4}{3 \pi r}$ and dielectric work $\varepsilon_{s p}(\omega)=\varepsilon_{R}+$ $I \varepsilon_{I}$, the productivity of termination is approximated by:

$$
Q_{e x t}=\frac{72 \pi r}{3 \lambda} \varepsilon_{e m}^{3 / 2} \frac{\varepsilon_{I}}{\left(\varepsilon_{R}+2 \varepsilon_{\text {em }}\right)^{2}+\varepsilon_{I}^{2}}
$$

The retention upgrade in metallic nano particle into a sun-based cell happens because of light dissipating and close field grouping of light that relies for the most part upon the molecule size, semiconductor ingestion and the electrical design of the sun powered cell. Metal nano particle are solid scattered of light at frequencies close to the Plasmon reverberation, because of a system wavering of the conduction electrons in the metal. For metal nanosphere molecule distance across $(\alpha)$ is considerably less than $1 /$ tenth frequency $(\lambda)$ of light, cross areas and productivity for dispersing, assimilation as given beneath:

$$
\left.\begin{array}{c}
C_{s c a}=\frac{1}{6 \pi}\left(\frac{2 \pi}{\lambda}\right)^{4}|\alpha|^{2} \\
C_{a b j}=\frac{2 \pi}{\lambda} \operatorname{lm}(\alpha) \\
Q_{s c a}=\frac{C_{s c a}}{\pi \alpha^{2}} \\
Q_{a b j}=\frac{C_{a b j}}{\pi \alpha^{2}}
\end{array}\right\}
$$

Upgrade happens under the condition that $\left|\varepsilon+2 \varepsilon_{\mathrm{m}}\right|$ is a least and at Plasmon reverberation recurrence, polarizability gets greatest as in (7). For a circle of volume $\mathrm{V}$ and dielectric work $\varepsilon$ $=\varepsilon_{1}+\mathrm{i} \varepsilon_{2}$. So, the cross-sectional annihilation is amount of dissipating and ingestion.

So, the cross-segment eradication and its effectiveness is given by:

$$
C_{\text {ext }}=9 \frac{\omega}{c} \varepsilon_{m}^{3 / 2} V \frac{\varepsilon_{2}}{\left[\varepsilon_{1}+2 \varepsilon_{m}\right]^{2}+\varepsilon_{2}^{2}} \text { and } Q_{\text {ext }}=\frac{C_{\text {ext }}}{\pi \alpha^{2}}
$$

The dispersing and ingestion of cross-areas rely upon polarizability $(\alpha)$ of the molecule is

$$
\alpha=4 \pi r^{3} \frac{\varepsilon_{\mathrm{sp}}(\omega)-\varepsilon_{\mathrm{em}}}{\varepsilon_{\mathrm{sp}}(\omega)+2 \varepsilon_{\mathrm{em}}}
$$

The retention, dissipating and annihilation efficiency versus episode frequency or wavelength for gold and with different diameter is appeared

The absorption, scattering and extinction efficiency versus wavelength for $\mathrm{Au}, \mathrm{Ag}, \mathrm{Cu}$ and $\mathrm{Al}$ with various diameters are shown in following figures bellow ${ }^{[7]}$ : 
Intensification and Optical Penetration in Plasmonic thin Film Solar Cell by Nano Particle on Metal Dielectric Semiconductor-Photonic Response
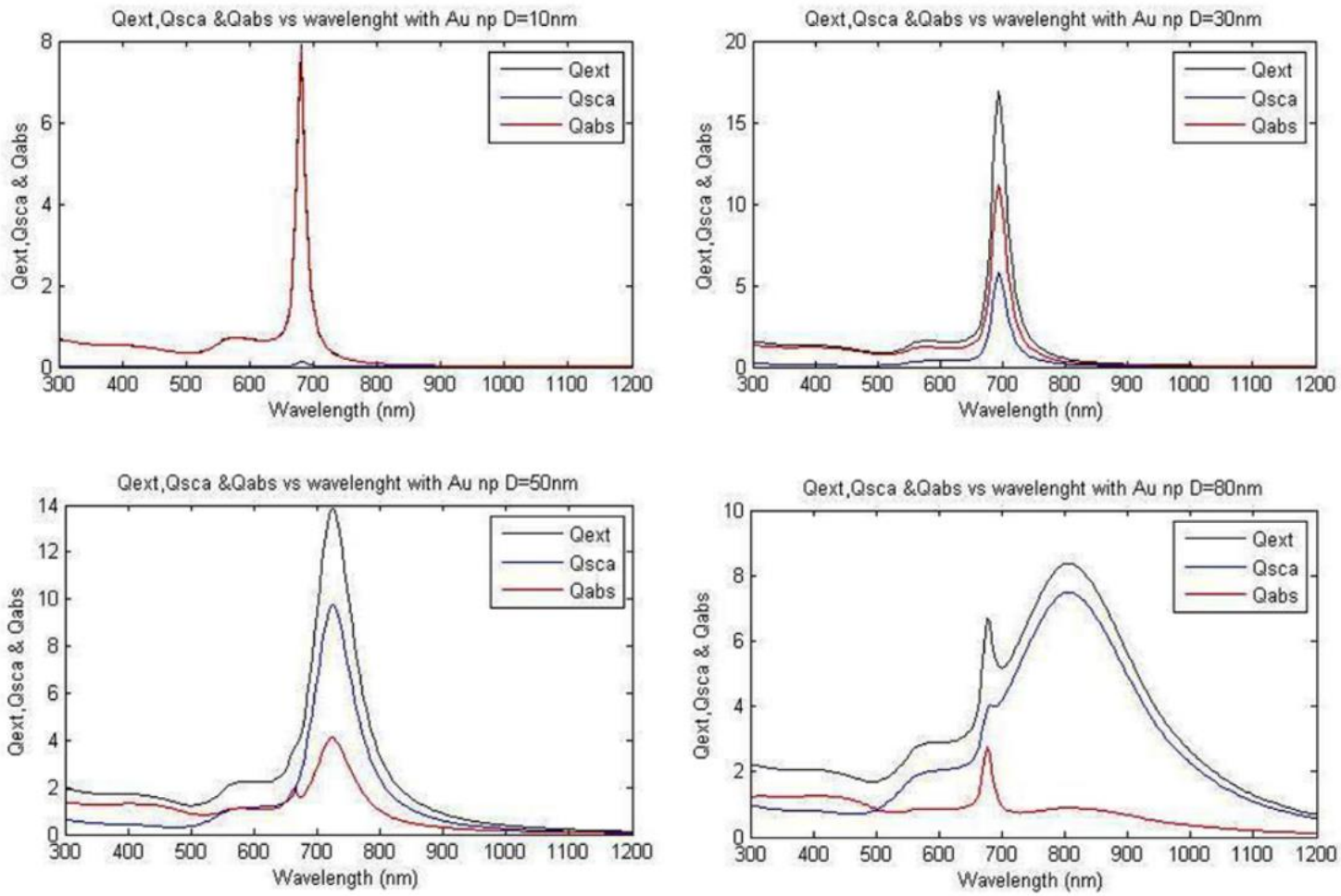

Figure 4 Absorption, scattering and extinction vs. wavelength for Au nanoparticle with $\mathrm{D}=10 \mathrm{~nm}, 30$ $\mathrm{nm}, 50 \mathrm{~nm}$ and $80 \mathrm{~nm}$
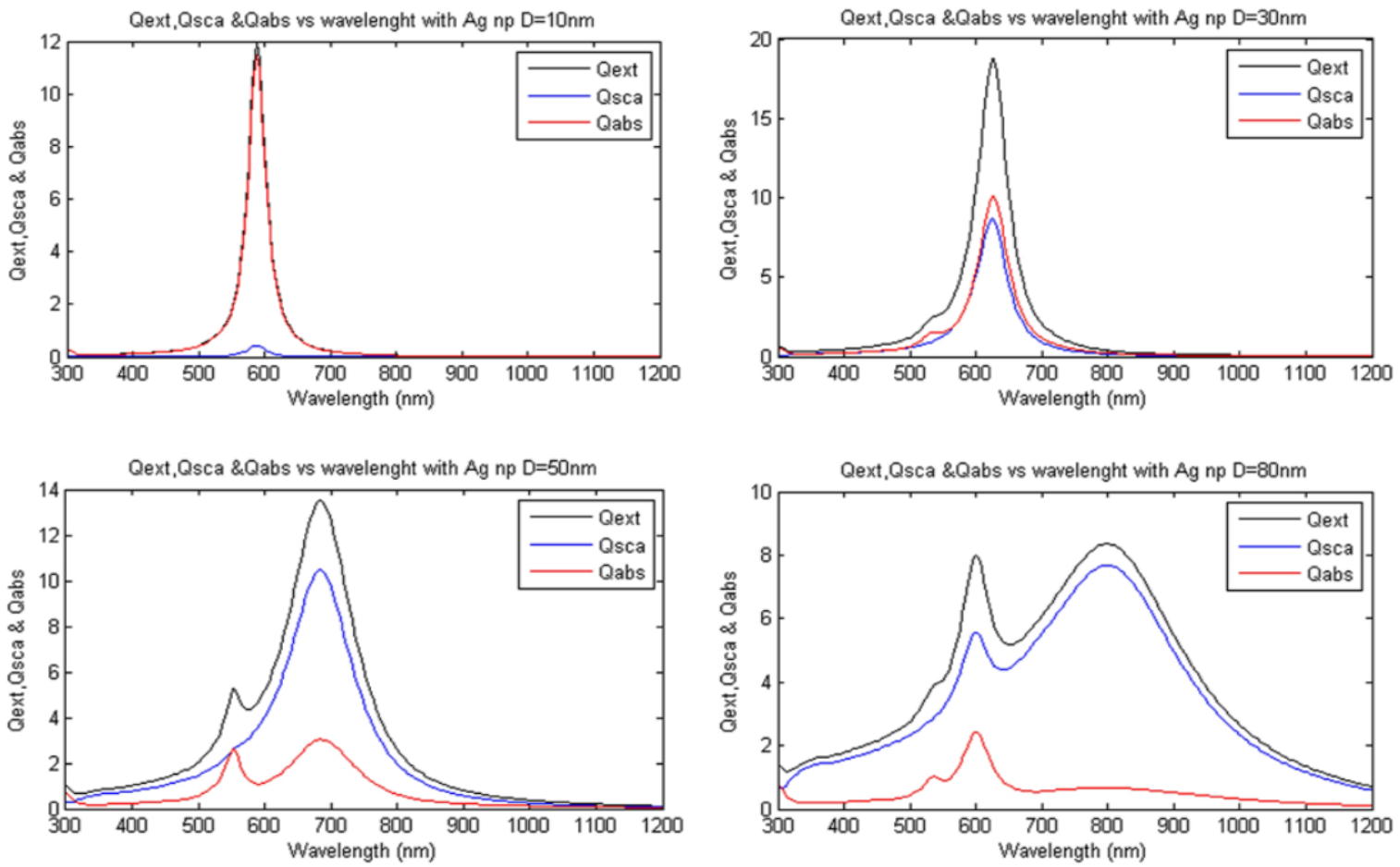

Figure 5 Absorption, scattering and extinction vs. wavelength for $\mathrm{Ag}$ nanoparticle with $\mathrm{D}=10 \mathrm{~nm}, 30$ $\mathrm{nm}, 50 \mathrm{~nm}$ and $80 \mathrm{~nm}$ 
Nabamitaa Roy, Priya Mahto, Samarpita Basu, Oudarjya Sen Sarma, Shreyasi Das and Saradindu Panda
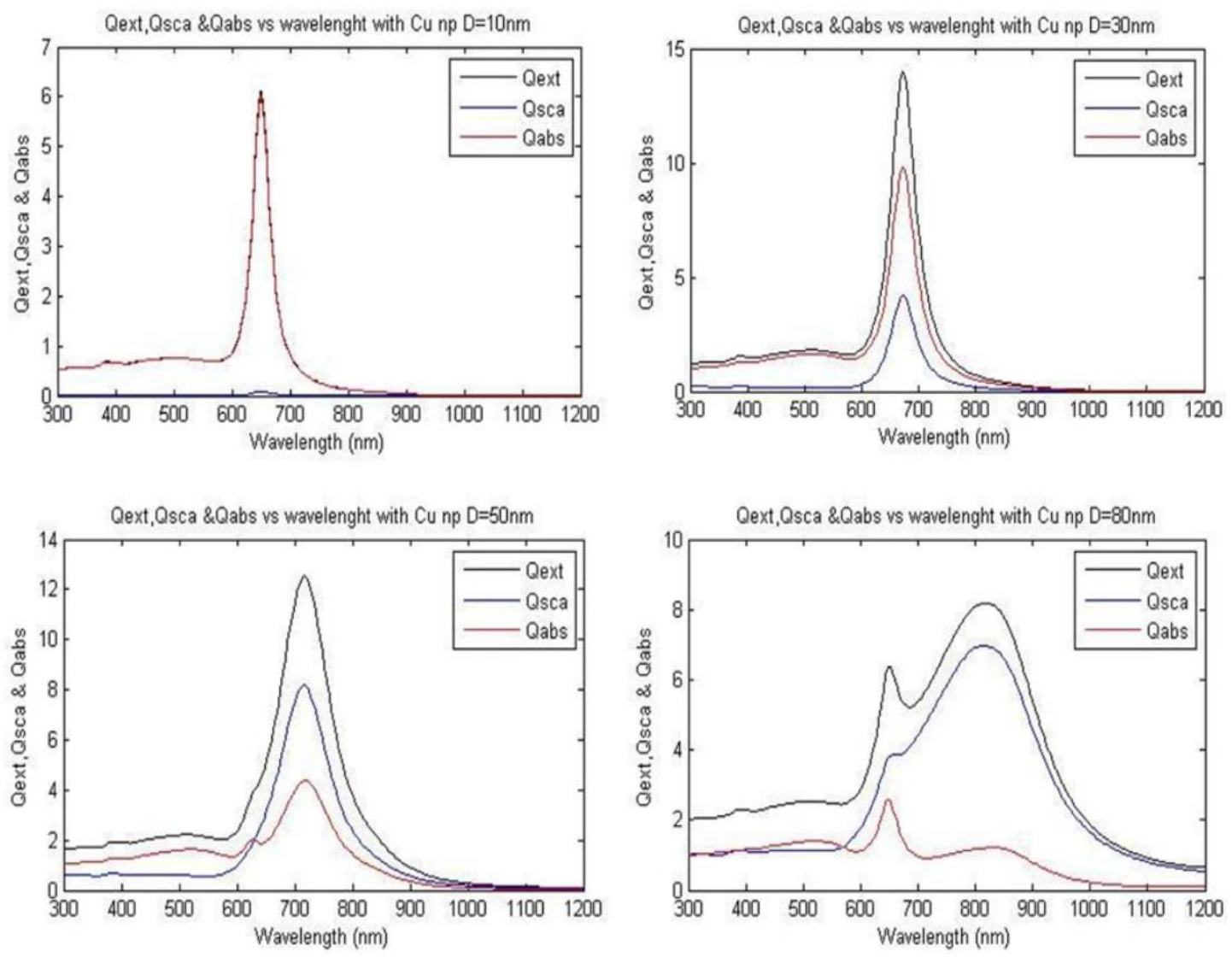

Figure 6 Absorption, scattering and extinction vs. wavelength for $\mathrm{Cu}$ nanoparticle with $\mathrm{D}=10 \mathrm{~nm}, 30$ $\mathrm{nm}, 50 \mathrm{~nm}$ and $80 \mathrm{~nm}$
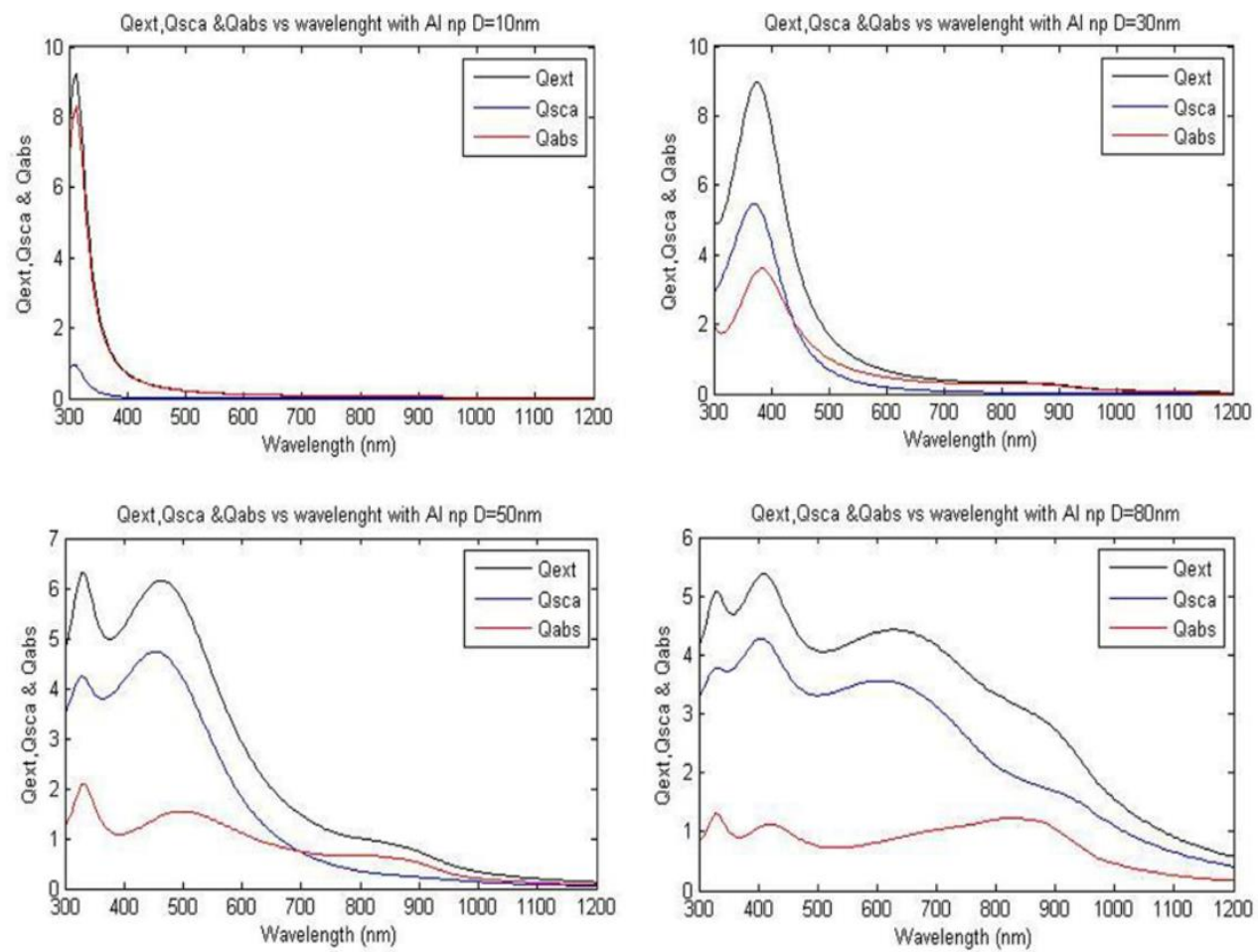

Figure 7 Absorption, scattering and extinction vs. wavelength for $\mathrm{Al}$ nanoparticle with $\mathrm{D}=10 \mathrm{~nm}, 30$ $\mathrm{nm}, 50 \mathrm{~nm}$ and $80 \mathrm{~nm}$ 


\subsection{FDTD Model ${ }^{[10]}$}

Here, we have contemplated the utilization of plasmonic nanostructures to improve sunlightbased cells for concentrating light catching. The commitment of every instrument depends generally on the molecule size, semiconductor assimilation and the electrical construction of the sunlight-based cell. In the finite-difference time domain (FDTD) model, we utilize limited contrast time area based and reenactment-based examination of the upgraded transmission of light into a slight $\mathrm{c}-\mathrm{Si}$ substrate by varieties of metal nano particles set on its top dielectric surface.

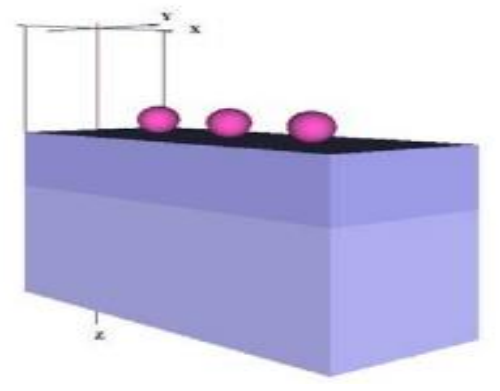

Figure 8 FDTD Model with Ag nano sphere

The FDTD model of proposed plasmonic slight film sunlight-based cell with Nobel metal nanoparticle at the highest point of dielectric layer. The model measurement is length $1000 \mathrm{~nm}$, width $425 \mathrm{~nm}$ and stature $425 \mathrm{~nm}$ with $\mathrm{Ag}$ nano sphere breadth $50 \mathrm{~nm}$. The episode power has been applied through $\mathrm{X}-\mathrm{Y}$ plane which is comparable to light occurrence at the AM1.5 worldwide sun powered range. The schematic investigation of Plasmon based light catching into a substrate by Nobel metal nanostructures put at the highest point of dielectric of the sun powered cell.

The various simulated fields components include Ex, Hy, Ez and pointing vector Sz form the observation plane 1 and observation plane 2 has studied.
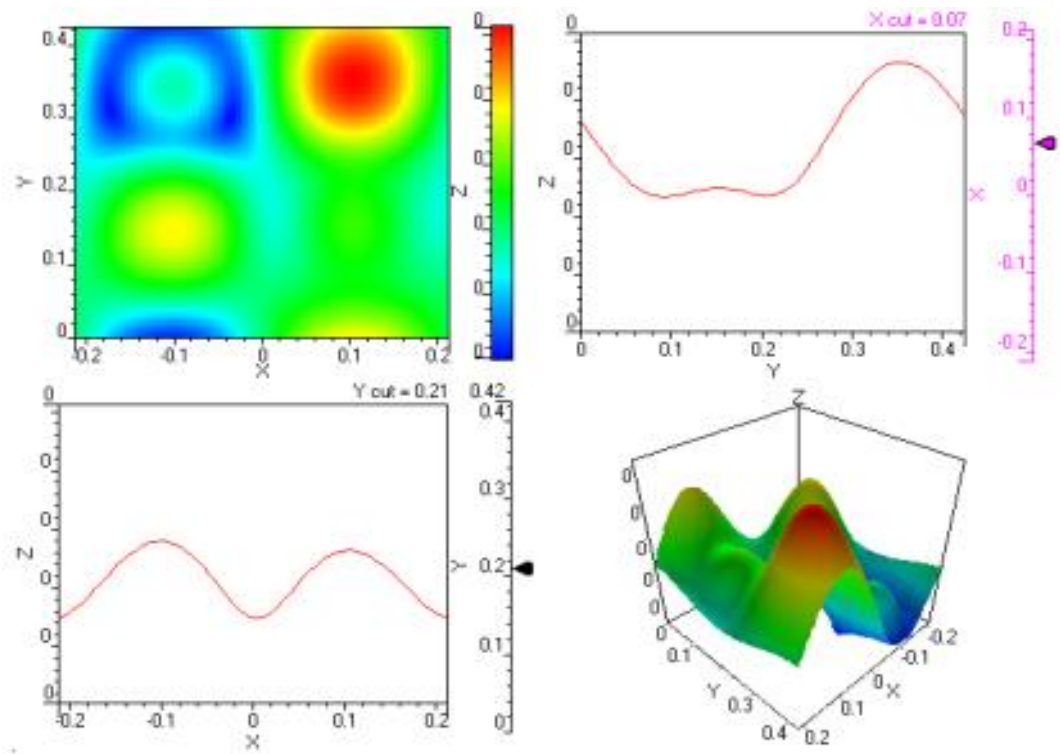

Figure 9 The simulated plot of fields of $E_{x}$, from observation plane 1 
Nabamitaa Roy, Priya Mahto, Samarpita Basu, Oudarjya Sen Sarma, Shreyasi Das and Saradindu Panda
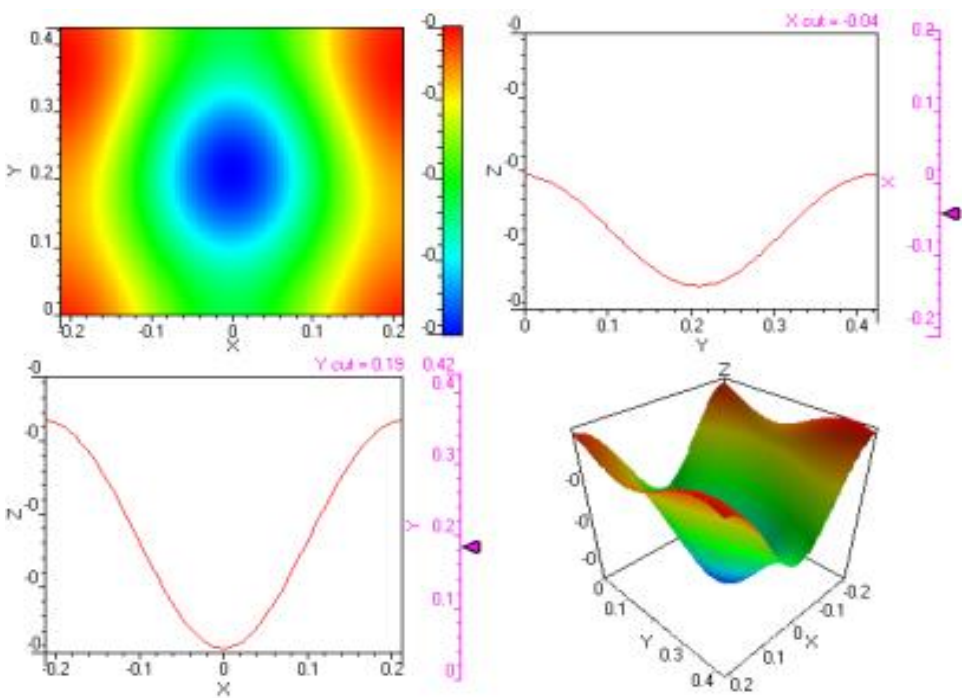

Figure 10 The simulated plot of pointing vector $\mathrm{S}_{\mathrm{z}}$ from observation plane 1
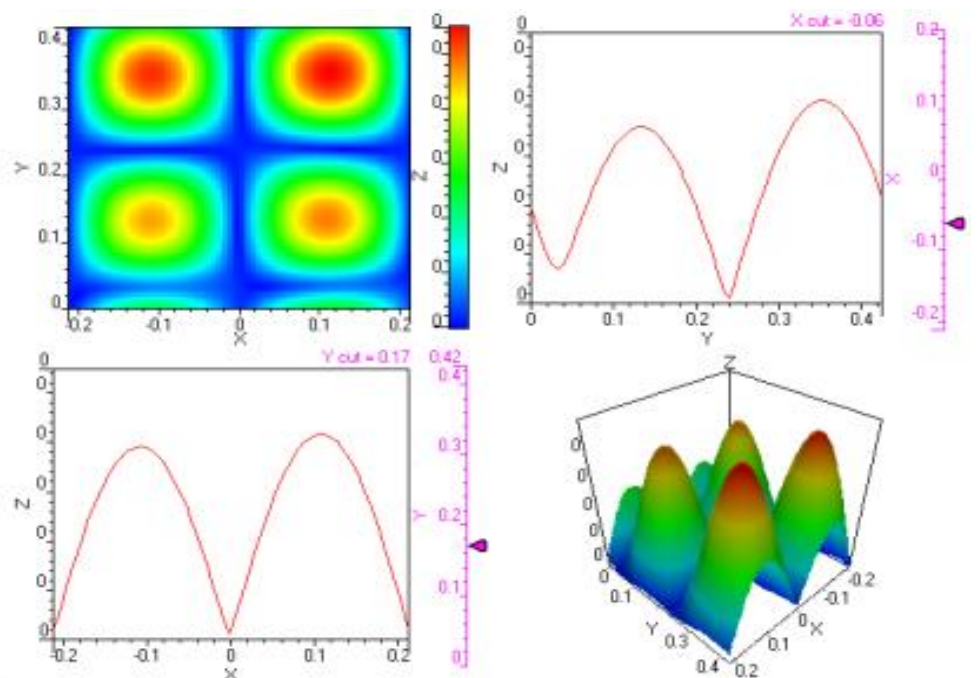

Figure 11 The simulated plot of fields of $\mathrm{H}_{\mathrm{y}}$, from observation plane 1
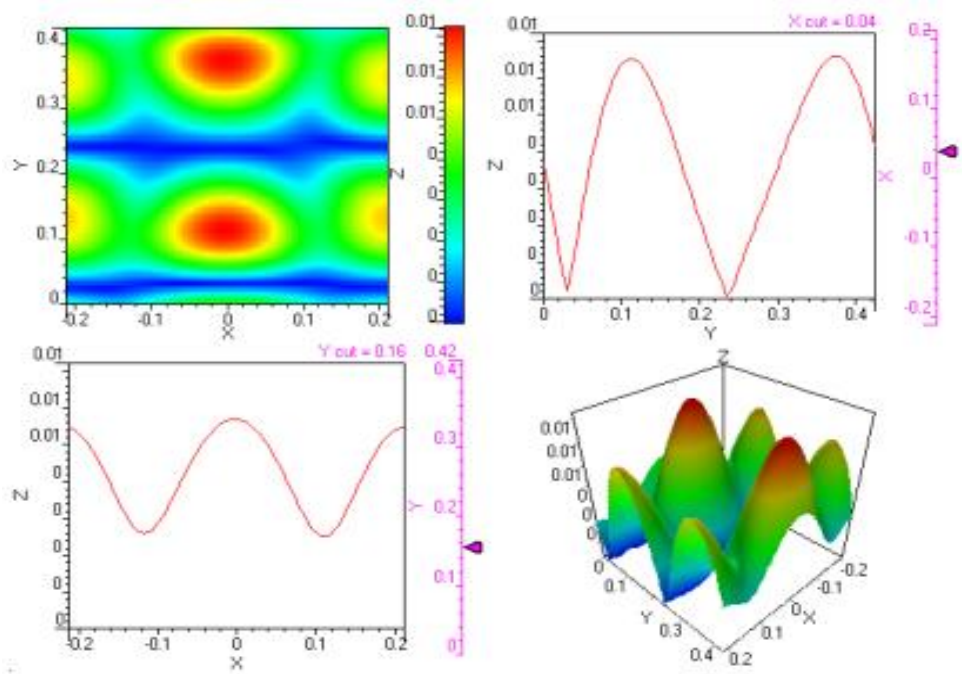

Figure 12 The simulated plot of fields of $E_{y}$, from observation plane 1 
Intensification and Optical Penetration in Plasmonic thin Film Solar Cell by Nano Particle on Metal Dielectric Semiconductor-Photonic Response

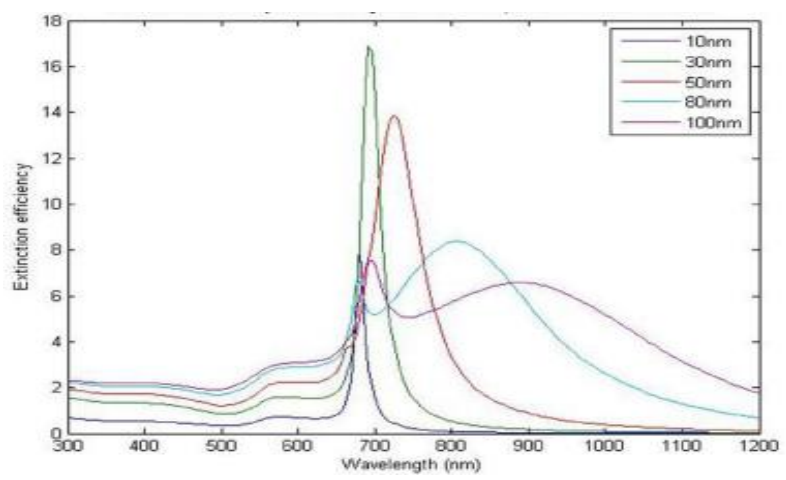

Figure 13 Extinction vs. wavelength for different radius of Au nanosphere

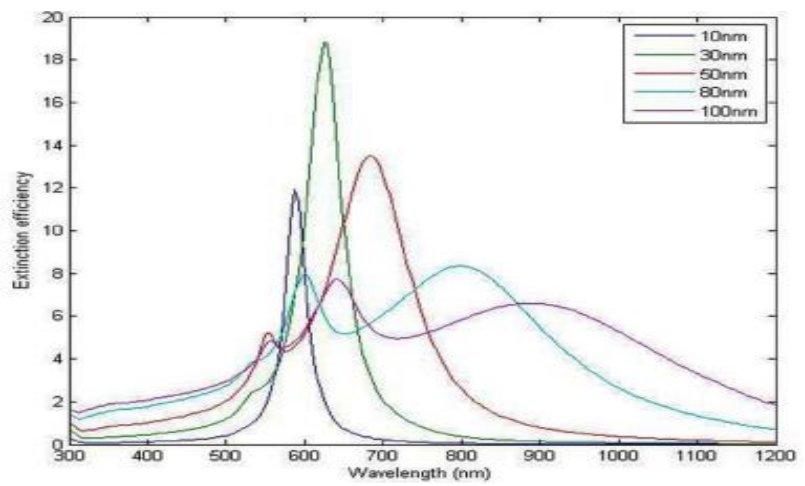

Figure 14 Extinction vs. wavelength for different radius of Ag nanosphere

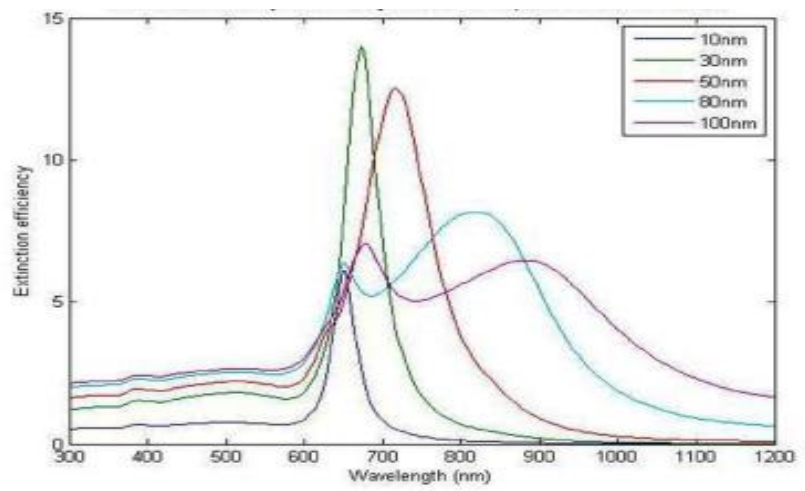

Figure 15 Extinction vs. wavelength for different radius of $\mathrm{Cu}$ nanosphere

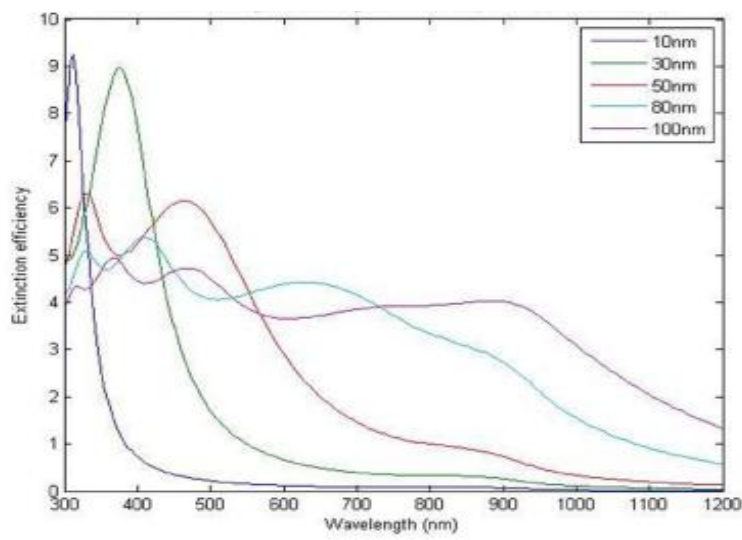

Figure 16 Extinction vs. wavelength for different radius of $\mathrm{Al}$ nanosphere 
Nabamitaa Roy, Priya Mahto, Samarpita Basu, Oudarjya Sen Sarma, Shreyasi Das and Saradindu Panda

Presently the near investigation of optical assimilation versus measurement for silver and gold nano sphere is appeared in Table $1 \&$ table 2.

Table 1 peak efficiency, wavelength, photon energy for Gold ( $\mathrm{Au}$ ) nanomaterial

\begin{tabular}{|l|l|l|l|}
\hline Radius & Peak efficiency (\%) & $\begin{array}{l}\text { Wavelength } \\
(\mathrm{nm})\end{array}$ & $\begin{array}{l}\text { Photon } \\
\text { energy(e v) }\end{array}$ \\
\hline 30 & 14.311 & 690 & 1.82 \\
\hline 50 & 17.141 & 720 & 1.69 \\
\hline 80 & 13.822 & 800 & 1.51 \\
\hline 100 & 7.501 & 696 & 1.80 \\
\hline
\end{tabular}

Table 2 peak efficiency, wavelength, photon energy for silver (Ag) nanomaterial

\begin{tabular}{|l|l|l|l|}
\hline Radius & Peak efficiency (\%) & $\begin{array}{l}\text { Wavelength } \\
(\mathrm{nm})\end{array}$ & $\begin{array}{l}\text { Photon } \\
\text { energy(e v) }\end{array}$ \\
\hline 20 & 18.511 & 600 & 2.07 \\
\hline 30 & 18.886 & 624 & 1.99 \\
\hline 50 & 13.412 & 680 & 1.80 \\
\hline 80 & 7.749 & 800 & 1.54 \\
\hline
\end{tabular}

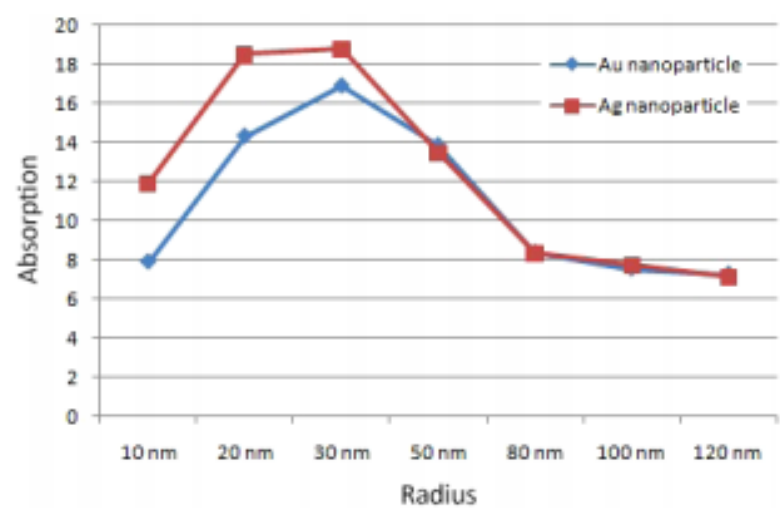

Figure 17 Graphical analysis of Radius versus Absorption

Additionally, we see that if the size of nanoparticle expands, the effectiveness will be forcefully decreased and the dissipating will be more noteworthy than assimilation as the size of nanoparticle increased and furthermore we see that if the range is under 50nm, ingestion turns out to be a lot higher than dispersing. Presently the relative investigation of optical assimilation versus measurement for silver and gold nanosphere is appeared in Figure. From this chart, we see that after $50 \mathrm{~nm}$ every one of the bends will unite. Since the molecule size is more noteworthy than $1 / 10$ th frequency of episode frequency ${ }^{[7]}$. In the present circumstance, front dispersing turns out to be more predominant than back dispersing.

\section{CONCLUSION}

From our investigation, if the size of the metallic nano sphere increments, at first the productivity increments up to a specific frequency then it turns out to be forcefully diminished. After $30 \mathrm{~nm}$, the dispersing gets higher than assimilation subsequently brings about diminished in photonic proficiency. At $10 \mathrm{~nm}$, silicon nitride performs better though at $30 \mathrm{~nm}$ aluminum oxide performs better compared to different dielectrics. Also, at $50 \mathrm{~nm}$ silicon dioxide performs better though at $100 \mathrm{~nm}$ aluminum oxide performs better compared to different dielectrics. The significant piece of the entire loss. In this way, the utilization of plasmonic might be a suitable strategy to control optical the field at the nano scales level to increment the photocurrent 
utilizing light catching and for upgrading productivity. In our FDTD-based sunlight-based cell model, the feigned result is portrayed by the field spread inside the sun powered cell because of the surface plasmon. From the feigned result, we seen that when the element of gold and silver nano spheres close to $30 \mathrm{~nm}$, the pinnacle optical efficiency regarding termination will be $16.89 \%$ and $18.78 \%{ }^{[8]}$, individually, because of the surface plasmon reverberation ${ }^{[9]}$. Additionally, if the measurement is higher than $50 \mathrm{~nm}$, the dispersing will overwhelm assimilation and is nearly indistinguishable for both $\mathrm{Ag}$ and $\mathrm{Au}$ and consequently there is a decline in efficiency. From our relative investigation, we see that the silver-based model gives a superior improvement than the gold-based model.

\section{REFERENCES}

[1] Sarkar, P. Panda, S; Maji, B. Mukhopadhyay. A. Kr. Study on surface Plasmon based improvement in absorption in plasmonic solar cell. International Journal of Nano particles, 2018, 10, 77-91. [http://dx.doi.org/10.1504/IJNP.2018.092678

[2] S. Enoch, N Bond, Plasmonic from Basics to Advanced Topics, Springer - Verlag, 2012

[3] A. Limmanee, T. Sugiura, H. Yamamoto, T. Sato, S. Miyajima, A. Yamada, M. Ko-nagai, Boron-doped Microcrystalline Silicon Oxide Film for Use as Back Surface Field in Cast Polycrystalline Silicon Solar Cells. Japanese Journal of Applied Physics, (2008), 47.

[4] K. A. Willets and R. P. Van Duyne, "Localized Surface Plasmon Resonance Spectroscopy and Sensing," Ann. Rev. Phys. chem, vol. 58, pp. 267-97, 2007

[5] S. Saga dev an, "Recent trends on Nanostructures based Solar energy application: A REVIEW, "Rev. Adv. Matter. Sci., vol. 34, pp. 44-61, 2013

[6] S. A. Maier, Plasmonic: Fundamentals and Applications, Springer, New York, 2007

[7] Sarkar, P.; Manna, A. Panda, S. Maji, B. Mukhopadhyay; A.Kr. Effect of nanoparticle size to improvement in absorption in Plasmonic solar cell. Materials Today: Proceedings, 2018, $5,21225-21231$.

[8] P.B. Johnson and R.W. Christy, "Optical constant of the Nobel metals," Physical Review B, vol. 6, num.12, 1972

[9] E. D. Paik, Handbook of Optical Constants of solids, Academic Press, 1985

[10] Sarkar, P.; Panda, S. Maji, B. Mukhopadhyay; A. Kr, Study on surface plasmon-based improvement in absorption in plasmonic solar cell, Int. J. Nanoparticles, Vol. 10, Nos. 1/2, 2018 